\title{
Acriflavine-resistant Mutants of Aspergillus nidulans
}

\author{
By J. A. ROPER AND ETTA KÄFER* \\ Department of Genetics, University of Glasgow
}

\begin{abstract}
SUMMARY : In three independently obtained mutant strains of Aspergillus nidulans, resistant in different degrees to acriflavine, resistance is due, in each case, to mutation in a single gene. Two of the mutant alleles, $A C R 1$ and $A C R 3$, are semi-dominant and either allele confers a high degree of resistance. These alleles are located about 23 units distal to the $w$ (white conidia) locus and are presumably allelic. A cross involving these two alleles in repulsion gave $0.1 \%$ sensitives. A third mutant allele (acr 2 ) is also located on the $w$ chromosome, but on the other arm about 25 units distal to the $a d 1$ locus and over 100 units distant from the $A C R 1$ and $A C R 3$ loci. This allele, which confers relatively slight resistance, is almost completely recessive. Diploid strains which carry any allele for resistance in heterozygous condition give, by vegetative segregation, haploid and homozygous diploid resistant types which are preferentially selected on medium with acriflavine. The use of this technique for the automatic selection of vegetative segregants provides an additional tool for analyses through the parasexual cycle.
\end{abstract}

Various mutant types have been used in genetic investigations with Aspergillus nidulans. Such types include those differing from wild type in conidial colour, colony morphology, nutritional requirements and ability to secrete certain enzymes (Pontecorvo, 1953). The present paper reports the extension of the range of mutant types to include those able to grow in the presence of certain substances which are inhibitory to the growth of the wild type. The aim in obtaining such resistant strains was that they should be used not only as a study in themselves, but also to provide an extra tool in the selection of vegetative segregants from heterozygous diploid strains. Strains of filamentous fungi carrying diploid nuclei give, though with a low frequency, mitotic segregants which result either from mitotic crossing-over (Pontecorvo \& Roper, 1952, 1953) or from haploidization (Pontecorvo \& Roper, 1953; Pontecorvo, Tarr Gloor \& Forbes, 1954). Genetic analyses based on the use of this parasexual process have been carried out in $A$. nidulans (Pontecorvo \& Roper, 1953; Pontecorvo et al. 1954; Pontecorvo \& Käfer, 1956), in A. niger (Pontecorvo, 1952; Pontecorvo, Roper \& Forbes, 1953) and in Penicillium chrysogenum (Pontecorvo \& Sermonti, 1954). The isolation and identification of segregants have been made in several ways: (i) visually, by isolating conidial heads of a colour different from those of the heterozygote (Pontecorvo \& Roper, 1953); (ii) nutritionally, by isolating segregants requiring some nutrient not required by the heterozygote (Forbes, 1952); (iii) nutritionally by the selection, through the use of suppressors, of segregants independent of some nutrient which the starting strain required (Pontecorvo \& Käfer, 1956);

* Present address : Biological Laboratories, Cold Spring Harbor, Long Island, N.Y., U.S.A. 
(iv) nutritionally by the selection of prototrophs from diploids carrying alleles in repulsion (Roper \& Pritchard, 1955). Only the last two of these techniques are automatic. It was hoped that either recessive or semi-dominant alleles conferring resistance would provide a further automatic technique of selection.

Aspergillus nidulans is surprisingly resistant to a wide range of substances which might be expected to be growth-inhibitory or toxic. Copper sulphate, organo-arsenical compounds and a wide range of dyestuffs even at high concentrations do not inhibit growth sufficiently to allow the selection of clearcut resistant strains. Of many substances tested acriflavine was the first to give, when used at workable concentration, effective inhibition of growth.

\section{METHODS}

The general techniques used throughout the present work were the same as those described by Pontecorvo (1953).

Media. Minimal medium (MM) Czapek-Dox with $2 \%$ glucose. Complete medium (CM), a complex medium containing yeast extract, casein hydrolysate, hydrolysed nucleic acid, vitamins, etc. Incubation was at $37^{\circ}$.

Organisms. Sensitive starting strains were selected from the laboratory stock of mutant strains. The symbols used to designate mutant alleles are those used previously (Pontecorvo, 1953). The mutant alleles used in this work, and the phenotypes these alleles determine, are listed in Table 1.

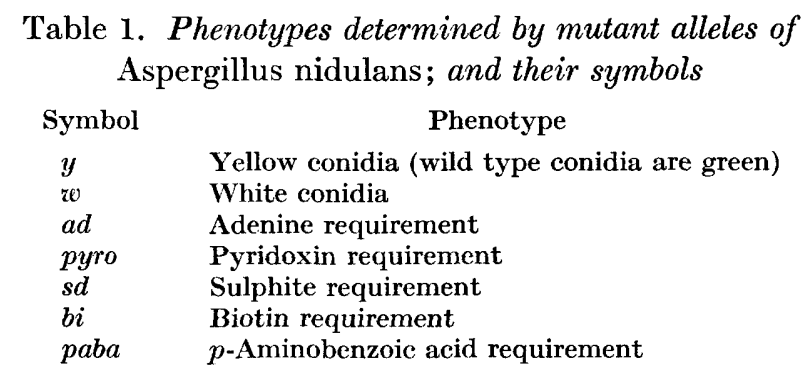

Isolation of mutants. Ten strains, carrying various mutant alleles, were selected from the laboratory stock and tested for sensitivity to acriflavine. Petri plates of CM with various concentrations of acriflavine were spread with conidial suspensions so that each plate had about $10^{7}$ conidia; these were examined after 3 days of incubation. All the ten strains were sensitive to acriflavine to approximately the same degree.

Mutants were selected by plating conidia of sensitive strains on CM containing $0.005 \%(\mathrm{w} / \mathrm{v})$ acriflavine. This concentration of acriflavine is approximately three times that required to prevent germination of sensitive conidia plated at a density of $10^{7}$ conidia/dish. In fact, at such high densities of conidia some diffuse growth was obtained round the edges of the dishes where masses of conidia had clumped. That this growth was due to adaptation and not mutation was shown by the transfer of conidia from such growth to further acriflavine medium on which it invariably failed to grow. The vigorously 
growing mutant types could be clearly distinguished from growth due to adaptation.

The first mutant was obtained from the plating of the strain pabal $y$; the second from ad15 paba $1 y$ and the third from ad1 pyro 4 sd bi 1. Although several resistant colonies were obtained at each plating only one was selected from each so as to avoid investigating a clone. Different starting strains in each case eliminated possible difficulties due to contamination. No attempt was made to estimate accurately the frequency of acriflavine-resistant mutants, but the yield of resistant colonies in each selection varied between 1 in $2 \times 10^{7}$ and 1 in $10^{8}$.

Detailed genetical analysis of the three selected resistant strains is described below. It was shown that in each strain a single mutant allele determined resistance. The three alleles were designated $A C R 1$, acr 2 and $A C R 3$.

\section{Degree of sensitivity of wild type and mutants}

Haploids. Sensitivity to acriflavine is not significantly affected by the concentration of vitamins or amino acids, and is independent of $\mathrm{pH}$ value within the range pH 4-7. However, sensitivity is dependent on the concentration of nucleic acid (cf. McIlwain, 1941). When the concentration of hydrolysed nucleic acid in the CM was increased 3- to 20-fold, resistance to acriflavine of both sensitives and resistants was increased about 3- to 8-fold. For this reason quantitative tests of sensitivity were made with standardized batches of CM.

Attempts to measure resistance by means of linear growth rate on different acriflavine concentrations are liable to considerable error owing to the relatively slow growth rate of this organism. Further, when this method was applied to heterozygotes, sectors, due to the selection of segregants (see below), made accurate measurement impossible.

To determine the resistance of a strain quantitatively, each of a series of plates of CM, with or without acriflavine, was spread with about 80 conidia. Ten replicates were used in each series so that for any one concentration of acriflavine 800 conidia of a strain were tested for ability to produce a colony after 3 days of incubation. Colony counts so obtained were expressed as a percentage of the counts on CM without acriflavine. Figure 1 shows the curves obtained for strains each carrying one of the mutant alleles $A C R 1$, acr 2 or $A C R 3$; wild type is shown for comparison. The degree of resistance conferred by each allele is different, though for general working purposes $A C R 1$ and $A C R 3$ are sufficiently similar to be interchangeable. That the differences in resistance were determined by the mutant alleles themselves and not by modifiers was shown by outcrossing and testing recombinants with each allele. Such recombinants always showed a degree of resistance not significantly different from that of the parent strain.

The three resistant strains also showed a greater resistance to crystal violet and malachite green than did wild type. The results with these two inhibitors were not always perfectly reproducible and resistance to them was not further investigated. 
Diploids. Using the technique of Roper (1952) 8 strains carrying diploid nuclei of the following genotypes with respect to acriflavine resistance were prepared: $A C R 1 / A C R 1, \operatorname{acr} 2 / \operatorname{acr} 2, A C R 3 / A C R 3, A C R 1 /+, \operatorname{acr} 2 /+$, $A C R 3 /+, A C R 1 / A C R 3$ and wild type. All the strains were also heterozygous for other markers, which determined nutritional requirements and conidial colour.

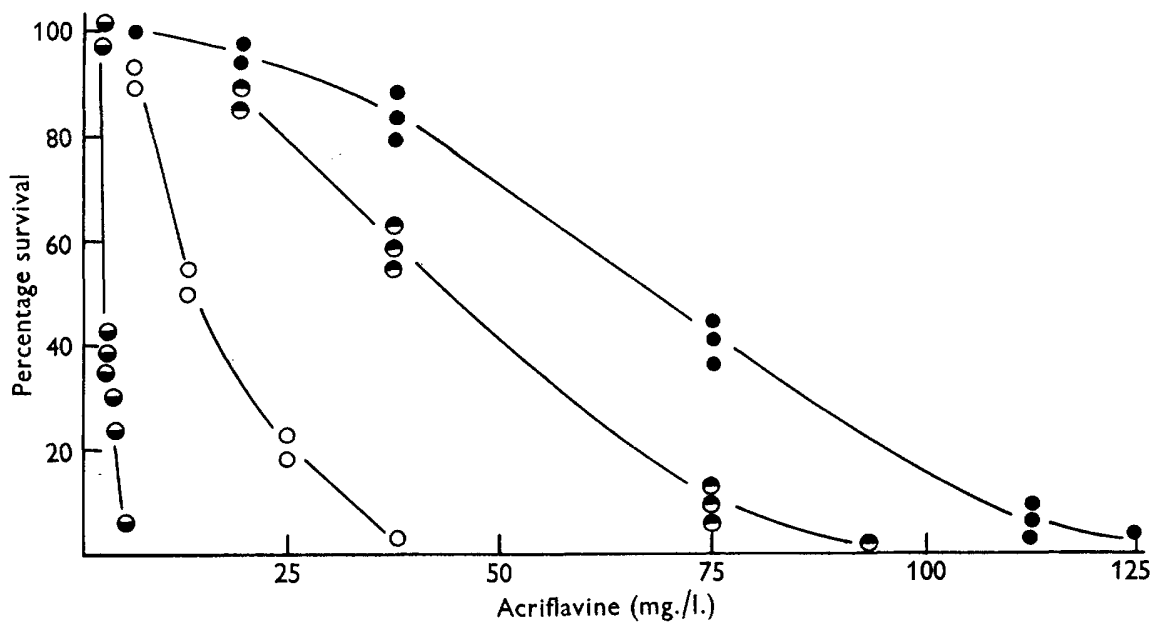

Fig. 1. Survival of conidia on CM with acriflavine. $\bullet, A C R 1 ; \ominus, A C R 3 ;$ O, acr $2 ; \ominus$, wild type.

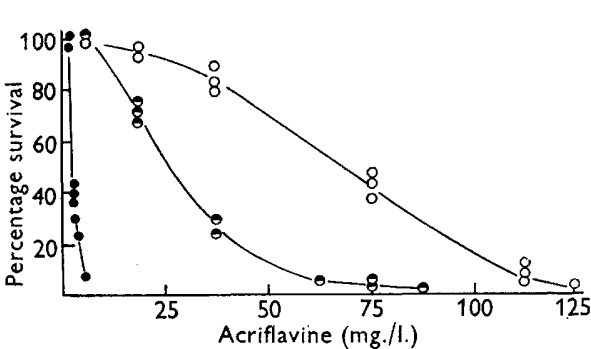

Fig. 2. Survival of conidia on $\mathrm{CM}$ with acriflavine. $O, A C R 1 ; \Theta, A C R 1 /+$; , wild type.

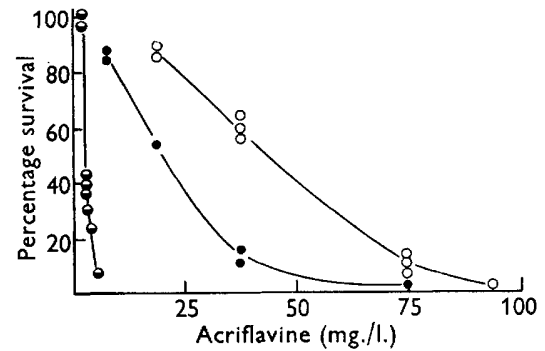

Fig. 3. Survival of conidia on $\mathrm{CM}$ with acriflavine. $\mathrm{O}, \mathrm{ACR} 3 ; \boldsymbol{O}, A C R 3 /+$; $\ominus$, wild type.

Each of the four strains $A C R 1 / A C R 1$, acr 2/acr $2, A C R 3 / A C R 3$ and the diploid wild type showed a resistance like that of the corresponding haploid. The resistance of the strains $A C R 1 /+, \operatorname{acr} 2 /+, A C R 3 /+$ and $A C R 1 / A C R 3$ are shown in Figs. 2-5. Figs. 2 and 3 show that the alleles $A C R 1$ and $A C R 3$ are semi-dominant; curves for the heterozygotes lie between those of wild type and the resistant homozygous diploid or the resistant haploid. At anything other than the lowest acriflavine concentrations the genotypes + (haploid or diploid), $A C R 1 /+$ and $A C R 1$ (or $A C R 1 / A C R 1$ ) can be distinguished not only in terms of viability but also by colony size (Pl. 1, fig. 1); 
the same applies to allele $A C R 3$. Not only that, but heterozygotes invariably sector (Pl. 1, fig. 2) on acriflavine medium to give sectors growing at the rate of the haploid or homozygous diploid resistant. Fig. 4 shows the behaviour of the strain $A C R 1 / A C R 3$ whose resistance lies between that of its components.

On the other hand, the strain acr $2 /+$ has very low resistance (Fig. 5) and can only be distinguished from wild type by careful tests of survival or the ability to produce resistant segregants. The allele acr $\mathbf{2}$ is almost completely recessive.

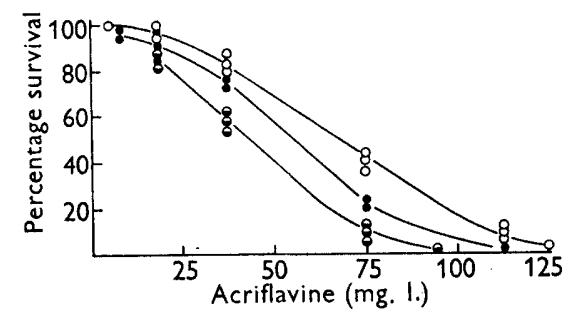

Fig. 4. Survival of conidia on CM with acriflavine. $O, A C R 1 ; \ominus, A C R 3$; ○, $A C R 1 / A C R 3$.

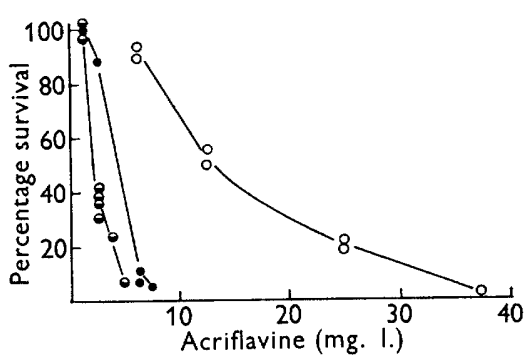

Fig. 5. Survival of conidia on $\mathrm{CM}$ with acriflavine. $\mathrm{O}, \operatorname{acr} 2 ; 0, \operatorname{acr} 2 /+$; $\ominus$, wild type.

Heterokaryons. A number of balanced heterokaryons were prepared which were comparable to certain of the above heterozygotes. So far this has been done only for the allele $A C R 1$. With respect to this allele the three investigated heterokaryons had nuclei of types: + with + ; with $A C R 1 ; A C R 1$ with $A C R$. The resistance of the heterokaryons could not be tested on the same basis as the resistance tests discussed above. Growth-rate measurements were made on gradient plates of MM (Szybalski, 1952) in which the lower layer contained acriflavine and the upper did not. Heterokaryons were subcultured by transfer to the gradient plates of hyphal tips from heterokaryotic colonies on minimal medium. Subculture was made to the point on the Szybalski layer plate where the acriflavine concentration was lowest. Such tests showed that the relative degrees of resistance of these three heterokaryons were comparable with the relative degrees of resistance of the corresponding diploids; that is, the allele $A C R 1$ is semi-dominant in a heterokaryon.

\section{Genetical analysis of the mutants}

Meiotic analysis. The determination of the genetical control of resistance and the location of the alleles involved was first made through the sexual cycle; routine techniques being used (Pontecorvo, 1953). From crosses of a sensitive strain to each resistant strain it was shown that mutation of a single gene determined resistance in each strain. The mutant alleles were designated $A C R 1, a c r 2$ and $A C R 3$. The allele $A C R 1$ was located $23 \cdot 0 \pm 1.9$ units from the $w$ locus. Subsequent mitotic analysis (see below) showed that this locus was distal to the $w$ locus. Similarly the allele $A C R 3$ was shown to be located in the same region. The allele acr 2 was located on the same chromosome 
$26.0 \pm 2.9$ units distal to the $a d 1$ locus; that is, more than 100 units from the $A C R 1$ and $A C R 3$ loci and on the other arm of that chromosome.

Physiological allelism of $A C R 1$ and $A C R 3$ could not be tested by the usual practice of observing the phenotype of the double heterozygote in trans arrangement since these mutant alleles are semi-dominant.

A cross was made involving the markers: $+A C R 1 \times w A C R 3$. Ascospores from the cross were plated on $\mathrm{CM}$ and colonies tested for resistance or sensitivity. Of 3468 colonies tested 4 ( 1 white conidia and 3 coloured conidia) were sensitive. Owing to the lack of closely linked markers it is impossible to say whether these sensitives resulted from recombination or reversion. In any case $A C R 1$ and $A C R 3$ are very closely linked if not, in fact, allelic.

Mitotic analysis with $A C R \mathrm{1}$ and $A C R 3$. When conidia of the heterozygote $A C R 1 /+$ or $A C R 3 /+$ were plated on media with appropriate concentrations of acriflavine the resulting colonies showed sectors after 2-4 days of incubation. Optimal conditions for selection of sectors was given by $\mathbf{C M}$ containing c. $\mathbf{0 . 0 0 2 5} \%(\mathrm{w} / \mathrm{v})$ acriflavine. Such sectors (Pl. 1, fig. 2) grew at the same rate as the haploid or homozygous diploid resistant types and were therefore presumed to be vegetative segregants of genotype $A C R$ or $A C R / A C R$. Segregants of such genotype should be able to outgrow the heterozygote on acriflavine medium. Isolates made from the tips of such sectors almost always gave pure cultures which could be immediately tested. Analysis of segregant sectors showed, as expected, that both haploid and diploid segregants arose (Pontecorvo \& Käfer, 1956). The data of these authors showed that from the heterozygotes $\frac{A C R w}{++} \bullet$ and $\frac{A C R+}{+w} \bullet \quad(\bullet=$ centromere) haploid segregants were white and coloured respectively.

From the former heterozygote the diploid segregants were, as expected, all $A C R / A C R$; some were $w / w$ and others $w /+$, showing that the order of the loci with respect to their centromere is as indicated.

Mitotic analysis with recessive allele acr 2 . Two techniques for segregant selection are possible with the recessive allele acr 2 . Some conidia formed by a strain $\operatorname{acr} 2 /+$ will have segregant nuclei of type acr 2 or acr $2 /$ acr 2 . These can be selected by plating large numbers of conidia formed by the heterozygote on CM containing $0.0013 \%(\mathrm{w} / \mathrm{v})$ acriflavine. Because of their clonal distribution some or even all of the segregants of any one culture of the heterozygote may be of common origin. For the analysis of segregants of certain independent origin, separate platings should be made, using in each case a different culture derived from a known heterozygous conidium. Only one resistant segregant should be taken from each plating. The selection method described above for the semi-dominant alleles $A C R 1$ and $A C R 3$ cannot be applied to the recessive allele $a c r 2$. No concentration of acriflavine has been found which is sufficiently low to permit enough growth of the acr $2 /+$ type for segregation to occur and yet is sufficiently high to select resistant segregants. However, this difficulty can be overcome by first growing the heterozygous types on medium without acriflavine. When growth is sufficient for segregation to have occurred acriflavine is added and the resistant segregants are selected; the technique is as 
follows. Conidia of the heterozygote acr $2 /+$ are plated so that each plate (diameter $9 \mathrm{~cm}$.) has about 10 conidia suspended in $7 \mathrm{ml}$. of $\mathrm{CM}$. When this layer is set a further layer of CM ( $5 \mathrm{ml}$./plate) is added. The dishes are then incubated for $24 \mathrm{hr}$., by which time the colonies are $4-8 \mathrm{~mm}$. in diameter. A top evenly-poured layer $(8 \mathrm{ml} . /$ plate) of $\mathbf{C M}$ containing $0.0013 \%(\mathrm{w} / \mathrm{v})$ acriflavine is then added and incubation continued. In the event that a conidium with a nucleus of genotype acr 2 or acr $2 /$ acr 2 was plated the whole colony fairly quickly grows through the acriflavine layer. Such colonies should be ignored as they represent segregation in the previous generation. In the case of a colony which originates from a heterozygous nucleus resistant sectors are seen after 2-4 days of further incubation. The sectors first appear as dense patches of growth (Pl. 1, fig. 3) some of which pierce the acriflavine layer. Most colonies show many sectors of clear clonal distribution; large sectors have their origin near the centre of the colony while the smaller sectors start from nearer the edge. After 6-8 days some of the sectors reach the surface and form conidia (Pl. 1, fig. 4). This technique has not yet been extensively applied and it is not excluded that a proportion of the sectors may arise by mutation. However, haploid segregants of expected genotype and diploid segregants have been obtained, and it seems likely that segregation and not mutation gives rise to the majority of sectors.

\section{DISCUSSION}

McIlwain (1941) showed that inhibition of bacterial growth by acriflavine was annulled by nucleotides with which acriflavine forms complex salts. $\mathrm{He}$ suggested that acriflavine inactivates enzyme systems of which nucleotides are an essential part. Ephrussi, Hottinguer \& Chimènes (1949) and Slonimski \& Ephrussi (1949) showed the inhibition by acriflavine of a particle-borne cytochrome system of yeast. The means by which gene-determined resistant strains arise is not yet known. In so far as nucleic acid or its components increases the acriflavine tolerance of both sensitive and resistant strains of Aspergillus nidulans the association between acriflavine inhibition and nucleic acid is again confirmed. These resistant strains provide a means for further investigation of the inhibitory action of acriflavine.

The semi-dominant alleles $A C R 1$ and $A C R 3$ determine qualitatively similar but quantitatively different phenotypes; they are an example of either allelic or closely-linked mutations which determine similar but not identical phenotypes. It is easily possible to make quantitative estimates of the degree of dominance or recessivity of each of the three mutant alleles and it should be possible to follow dominance modification under conditions favouring such modification.

For the present, the resistant strains have been exploited mainly for use in the parasexual cycle. The semi-dominant and recessive alleles provide a technique for the automatic selection of vegetative segregants of independent origin. The rate of sectoring of heterozygotes gives a relative, though not absolute, estimate of rates of segregation and opens a way for the investigation of genetical and environmental factors which affect this rate. For use in the parasexual cycle the ideal would be to have at least one distal mutant 
Journal of General Microbiology, Vol. 16, No. 3
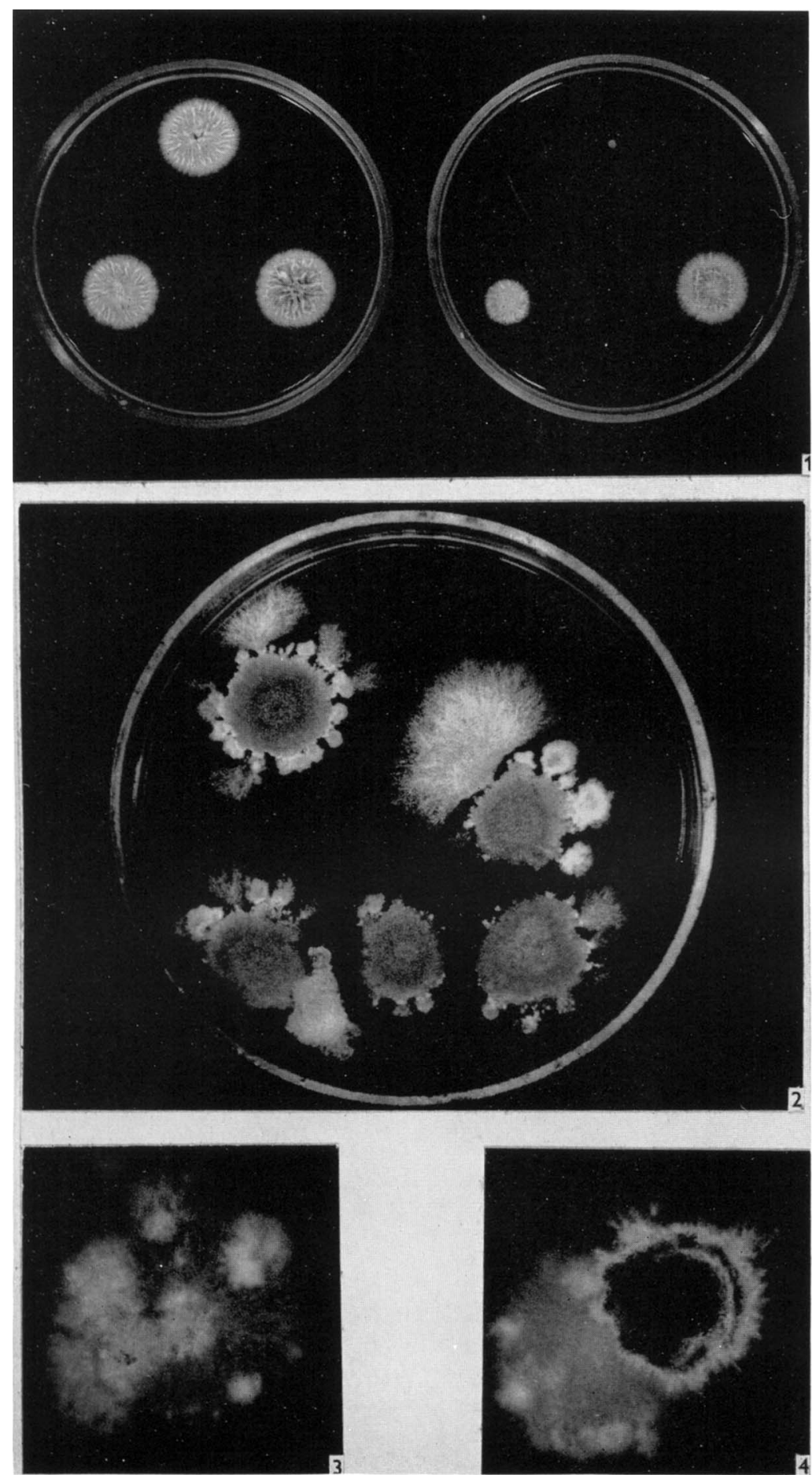

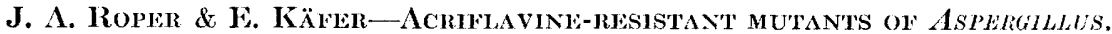
P'LATE 1 
allele, which confers resistance to one or other substance, on each chromosome arm of the whole chromosome complement. In the case of fungi which have multinucleate conidia the selection of segregants by means of recessive visible markers is likely to be inefficient since only groups of homokaryotic mutant conidia will be detected. Essentially the same applies to those fungi in which the conidial colour markers are non-autonomous. Selection through resistance as described in this account should provide one means of overcoming this difficulty.

The authors are indebted to Professor G. Pontecorvo, F.R.S., for advice and criticism both during the investigations reported and in the preparation of this account.

The work reported here is part of a programme supported by the Nuffield Foundation.

\section{REFERENCES}

Ephrussi, B., Hottinguer, H. \& Chimènes, A. (1949). Action de l'acriflavine sur les levures. Ann. Inst. Pasteur, 76, 351.

Forbes, E. (1952). The use of $\mathrm{SO}_{2}$ for selecting auxotrophs in filamentous fungi. Microbial genet. Bull. 6, 26.

McIlwaIn, H. (1941). A nutritional investigation of the antibacterial action of acriflavine. Biochem. J. 35, 1311.

Ponteconvo, G. (1952). Non-random distribution of multiple mitotic crossing-over among nuclei of heterozygous diploid Aspergillus. Nature, Lond. 170, 204.

Ponteconvo, G. (1953). The genetics of Aspergillus nidulans. Advanc. Genet. 5, 141.

Ponteconvo, G. \& KäFer, E. (1956). Mapping the chromosomes by means of mitotic recombination. Proc. R. phys. Soc. Edinb. 25, 16.

Pontecorvo, G. \& Roper, J. A. (1952). Genetic analysis without sexual reproduction by means of polyploidy in Aspergillus nidulans. J. gen. Microbiol. 6, vii.

Pontecorvo, G. \& Roper, J. A. (1953). Diploids and mitotic recombination. Advanc. Genet. 5, 218.

Pontecorvo, G., Roper, J. A. \& Forbes, E. (1953). Genetic recombination without sexual reproduction in Aspergillus niger. J. gen. Microbiol. 8, 198.

Ponteconvo, G. \& Sermonti, G. (1954). Parasexual recombination in Penicillium chrysogenum. J. gen. Microbiol. 11, 94.

Pontecorvo, G., TArr Gloon, E. \& Forbes, E. (1954). Analysis of mitotic recombination in Aspergillus nidulans. J. Genet. 52, 226.

Roper, J. A. (1952). Production of heterozygous diploids in filamentous fungi. Experientia, 8, 14.

Roper, J. A. \& Pritchard, R. H. (1955). Recovery of the complementary products of mitotic crossing-over. Nature, Lond. 175, 639.

Slonimski, P. \& Epiruussi, B. (1949). Action de l'acriflavine sur les levures. V. Le système des cytochromes des mutants 'petite colonies'. Ann. Inst.Pasteur, 77, 47.

Szybalski, W. (1952). Gradient-plate technique for study of bacterial resistance. Science, 116, 46.

\section{EXPLANATION OF PLATE}

Fig. 1. Growth of the strains $A C R 1$ (right), $A C R 1 /+$ (left) and wild type (top) on CM (left plate) and CM with $0.005 \%(\mathrm{w} / \mathrm{v})$ acriflavine (right plate).

Fig. 2. Resistant sectors formed by strain $A C R 1 /+$ on CM with $0.0025 \%(\mathrm{w} / \mathrm{v})$ acriflavine.

Fig. 3. Resistant sectors formed by strain acr $2 /+$ on $\mathrm{CM}$ with acriflavine. The sectors have not yet reached the surface of the medium. $\times 3$.

Fig. 4. A resistant sector from strain acr $2 /+$ after 7 days of incubation. The sector has reach ${ }^{\circ}$ the surface and formed conidia. $\times 3$.

(Received 10 December 1956) 